\title{
Stability Analysis of a Type of T-S Fuzzy Control Systems Using Off-Axis Circle Criterion
}

\author{
Kairui Cao* \\ Center for Control Theory and Guidance Technology \\ Harbin Institute of Technology, Harbin 150001, China \\ E-mail: kcaohit@gmail.com \\ X. Z. Gao ${ }^{1,2}$ \\ ${ }^{1}$ College of Information Engineering \\ Shanghai Maritime University \\ 1550 Pu Dong Da Dao, Shanghai 200135, P. R. China \\ ${ }^{2}$ Department of Automation and Systems Technology \\ Aalto University School of Electrical Engineering \\ FI-00076 Aalto, Finland \\ E-mail:xiao-zhi.gao@aalto.fi \\ Xianlin Huang \\ Center for Control Theory and Guidance Technology \\ Harbin Institute of Technology, Harbin 150001, China \\ E-mail: xlinhuang@hit.edu.cn \\ Xiaojun Ban \\ Center for Control Theory and Guidance Technology \\ Harbin Institute of Technology, Harbin 150001, China \\ E-mail: banxiaojun@hit.edu.cn \\ Received 3 September 2010 \\ Accepted 15 May 2012
}

\begin{abstract}
In this paper, based on the off-axis circle criterion, a sufficient condition with a simple graphical explanation is derived to analyze the global asymptotic stability of a type of Takagi-Sugeno (T-S) fuzzy control systems in case of different constant reference inputs. Three numerical examples are given to demonstrate how to use the proposed method in analyzing the T-S fuzzy control systems.
\end{abstract}

Keywords: T-S fuzzy control systems, stability analysis, circle criterion, off-axis circle criterion.

\section{Introduction}

In the history of fuzzy control theory, the TakagiSugeno (T-S) fuzzy model ${ }^{1}$ is a famous landmark. For a given T-S fuzzy model, Sugeno and Kang propose an interesting fuzzy controller design approach named Parallel Distributed Compensation ${ }^{2}$. The corresponding stability analysis is given in Ref. 3. Furthermore, more relaxed sufficient stability conditions have been obtained to reduce their conservatism. For example, a relaxed condition is derived based on piecewise quadratic Lyapunov functions in Ref. 4. Feng presents a comprehensive survey on the recent progresses in the stability analysis issues of the T-S fuzzy systems ${ }^{5}$. Based on these stability and robust stability analysis, numerous systematic design schemes have been proposed to synthesize the T-S fuzzy controllers in order to guarantee the stability or performances of the

${ }^{*}$ Corresponding address: P. O. Box 3032, Yikuang Street 2\#, Nangang District, Harbin 150001, China. 
overall T-S fuzzy control systems even in the presence of parameter uncertainties ${ }^{6,7,8}$. Most of the above work is discussed only in the time domain, and the results are mainly presented in the form of Linear Matrix Inequalities (LMIs). Some numerical optimization methods, such as interior point method, are utilized to solve the LMIs. With the popular MATLAB LMI tool box, the system analysis and controller design can be made in an efficient way. However, to some extent, the LMI-based techniques heavily depend on numerical solutions. Thus, it is indeed of great significance to find alternative techniques to analyze the T-S fuzzy control systems, which should be more straightforward as well as less numerically dependent.

As we know that the frequency response methods have been well developed and widely used by control engineers during the past decades. For example, the effect of noise in a control system can be evaluated by its frequency response. One of the most prominent features of the frequency domain-based methods is that some useful graphs, e.g., Bode and Nyquist plots, can be employed to analyze the performances of the closedloop systems or synthesis the controllers for certain plants. Since the T-S fuzzy model usually consists of a family of local linear dynamic systems, it is valuable to graphically analyze the T-S fuzzy systems in the frequency domain. As a matter of fact, the work of exploring the fuzzy control systems in the frequency domain can be dated to Ref. 9, in which the describing function method is used to analyze the Mamdani type fuzzy control systems. More relevant references can also be found ${ }^{10}$. The circle criterion is applied for the stability analysis of the Mamdani type fuzzy control systems ${ }^{11,12}$. For the recent contributions, interested readers can refer to Refs. 13, 14, 15, and references therein, in which different kinds of Mamdani type fuzzy control systems are considered.

A lot of similar work has also been done for the T-S fuzzy control systems. For example, the describing function method is applied in stability analysis ${ }^{16}$. The circle criterion is employed to analyze the stability of TS fuzzy control systems in ${ }^{17}$. Unfortunately, there is no corresponding graphical interpretation in the frequency domain yet. Although the circle criterion with its graphical interpretation is used to deal with the issue of robust stability for the T-S fuzzy controller 18, 19, however, this fuzzy controller is only one special kind of nonlinear controller, and the available results cannot be directly generalized to analyze the general fuzzy control systems. In our previous work, the circle criterion is deployed to derive sufficient stability conditions for the T-S fuzzy control systems with a graphical interpretation in the senses of Lyapunov stability ${ }^{20,21}$. The off-axis circle criterion ${ }^{22}$ is also employed to analyze the same type of T-S fuzzy control systems ${ }^{23}$. Nevertheless, the reference inputs of all the aforementioned fuzzy control systems are always assumed to be zero. It is well known that the reference inputs, in fact, have a significant impact on the stability of the T-S fuzzy control systems. The aim of this paper is to generalize the off-axis circle criterion-based stability condition to the T-S fuzzy control systems in case of different constant reference inputs. The advantage of our method over the existing circle criterion criterion-based approach ${ }^{11,20}$ is that it can still guarantee the asymptotic stability under the above circumstances.

The rest of this paper is organized as follows. Section 2 defines the T-S fuzzy control system to be studied. In Section 3, a typical case study is illustrated to show the influences of the reference inputs on the stability of the fuzzy control systems. Section 4 presents the main results of the paper: a new stability condition with graphical interpretation is proposed for the T-S fuzzy control systems by using the off-axis circle criterion. Our method can guarantee the global asymptotic stability of the T-S fuzzy control systems in case of different constant reference inputs. Three simulation examples are provided to demonstrate how to use the proposed approach for the stability analysis in Section 5 . Some remarks and conclusions are finally drawn in Section 6.

\section{The T-S Fuzzy Control System}

The structure of the T-S fuzzy control system studied in this paper is shown in Fig. 1, where FLC and $G(s)$ are the T-S fuzzy controller and a linear Single Input Single Output (SISO) plant, respectively, $r$ is the reference input, and $u$ and $y$ are the outputs of the FLC and plant, respectively.

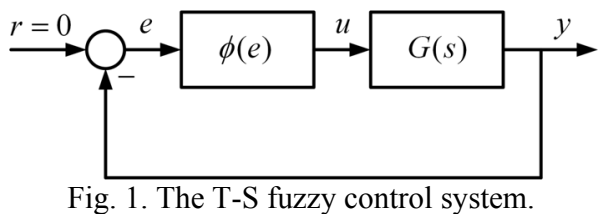


This T-S fuzzy controller consists of reasoning rules, and the $i$-th rule is of the following form:

Rule $i$ : If $e$ is $M_{i}$, then $u_{i}=k_{i} e, i=1, \cdots, n$, (1) where $e$ is the input of the fuzzy controller, $u_{i}$ is the output of the local consequent function, which is actually a proportional controller, and $M_{i}$ is the fuzzy set, whose corresponding membership function is denoted by $\mu_{i}$. The membership functions are illustrated in Fig. 2, where $a, b>0$.

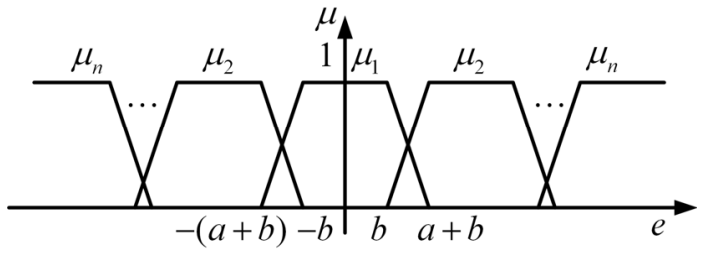

Fig. 2. Membership functions of $M_{i}$.

If $\phi(e)$ represents the nonlinear function achieved by the T-S fuzzy controller shown in Fig. 1, for the simplification of our presentation, we conclude that $\phi(e)$ belongs to the class $M_{\left[k_{\min }, k_{\max }\right]}$, if

$$
k_{\min } \leq \frac{\phi\left(e_{2}\right)-\phi\left(e_{1}\right)}{e_{2}-e_{1}} \leq k_{\max }, e_{1} \neq e_{2} .
$$

Lemma 1: If $\phi(e)$ represents the nonlinear function achieved by the T-S fuzzy controller in Fig. 1, and let

$$
\begin{gathered}
A_{i}=\left\{k_{i}, \frac{(i-1) a+(2 i-1) b}{a}\left(k_{i+1}-k_{i}\right)+k_{i},\right. \\
\left.\frac{(i-1) a+(2 i-1) b}{a}\left(k_{i+1}-k_{i}\right)+\left(2 k_{i+1}-k_{i}\right)\right\}, i<n, \\
A_{n}=\left\{k_{n}\right\}, \\
A=\bigcup_{i=1}^{n} A_{i},
\end{gathered}
$$

and denote

$$
\begin{aligned}
& k_{\text {min }}=\min \{z, z \in A\}, \\
& k_{\text {max }}=\max \{z, z \in A\} .
\end{aligned}
$$

Then, we have $\phi(e) \in M_{\left[k_{\min }, k_{\max }\right]}$.

Proof. With the fuzzy logic rules (1) and membership functions in Fig. 2, it is obvious that the mathematical expression of $\phi(e)$ is continuous, and the problem of finding the maximum and minimum of $\frac{\phi\left(e_{2}\right)-\phi\left(e_{1}\right)}{e_{2}-e_{1}}$ for all $e_{1} \neq e_{2}$ is equivalent to finding the maximum and mini $\neg$ mum of the derivative of $\phi(e)$. As $\phi(e)$ is odd, we only focus on $e \geq 0$ here.

When $0 \leq e<a+b$,

$$
\begin{gathered}
\phi(e)=\left\{\begin{array}{c}
k_{1} e, 0 \leq e<b ; \\
\frac{1}{a}\left(k_{2}-k_{1}\right) e^{2}-\frac{b}{a}\left(k_{2}-k_{1}\right) e \\
+k_{1} e, b \leq e<a+b .
\end{array}\right. \\
\dot{\phi}(e)=\left\{\begin{array}{c}
k_{1}, 0 \leq e<b ; \\
\frac{2}{a}\left(k_{2}-k_{1}\right)-\frac{b}{a}\left(k_{2}-k_{1}\right)+k_{1}, b \leq e<a+b .
\end{array}\right.
\end{gathered}
$$

We can observe that both $k_{1}$ and $-\frac{b}{a}\left(k_{2}-k_{1}\right)+k_{1}$ are constants. Since

$$
\begin{gathered}
\left.\dot{\phi}(e)\right|_{e=b^{-}}=\frac{b}{a}\left(k_{2}-k_{1}\right)+k_{1}, \\
\left.\dot{\phi}(e)\right|_{e=(a+b)^{+}}=\frac{b}{a}\left(k_{2}-k_{1}\right)+2 k_{2}-k_{1},
\end{gathered}
$$

the maximum and minimum of $\dot{\phi}(e)$ must be in the set

$$
A_{1}=\left\{k_{1}, \frac{b}{a}\left(k_{2}-k_{1}\right)+k_{1}, \frac{b}{a}\left(k_{2}-k_{1}\right)+2 k_{2}-k_{1}\right\} .
$$

When $a+b \leq e<(n-1) a+(2 n-3) b$, there exists an integer $1<i<n$, such that

$$
e \in[(i-1) a+(2 i-3) b, i a+(2 i-1) b) .
$$

We can get

$$
\phi(e)=\left\{\begin{array}{c}
k_{i} e,(i-1) a+(2 i-3) b \leq e<(i-1) a+(2 i-1) b ; \\
\frac{1}{a}\left(k_{i+1}-k_{i}\right) e^{2}-\frac{(i-1) a+(2 i-1) b}{a}\left(k_{i+1}-k_{i}\right) e \\
\quad+k_{i} e,(i-1) a+(2 i-1) b \leq e<i a+(2 i-1) b .
\end{array}\right.
$$

$$
\dot{\phi}(e)=\left\{\begin{array}{c}
k_{i},(i-1) a+(2 i-3) b \leq e<(i-1) a+(2 i-1) b ; \\
\frac{2}{a}\left(k_{i+1}-k_{i}\right) e-\frac{(i-1) a+(2 i-1) b}{a}\left(k_{i+1}-k_{i}\right) \\
\quad+k_{i},(i-1) a+(2 i-1) b \leq e<i a+(2 i-1) b .
\end{array}\right.
$$

Because

$$
\left.\dot{\phi}(e)\right|_{e=[(i-1) a+(2 i-1) b]^{-}}=\frac{(i-1) a+(2 i-1) b}{a}\left(k_{i+1}-k_{i}\right)+k_{i},
$$

$$
\left.\dot{\phi}(e)\right|_{e=[i a+(2 i-1) b]^{-}}=\frac{(i-1) a+(2 i-1) b}{a}\left(k_{i+1}-k_{i}\right)+2 k_{i+1}-k_{i} \text {. }
$$

Then the maximum and minimum of $\dot{\phi}(e)$ must be in $A_{i}$.

When $(n-1) a+(2 n-3) b \leq e$, then $\phi(e)=k_{n} e$, and there is

$$
\dot{\phi}(e)=k_{n} .
$$


From the above analysis, it is apparent that the maximum and minimum of $\dot{\phi}(e)$ in the domain $R$ are in the set $A=\bigcup_{i=1}^{n} A_{i}$. Thus, $\phi(e) \in M_{\left[k_{\min }, k_{\max }\right]}$.

\section{A Case Study of the Influences of Reference Inputs}

Consider a simple T-S fuzzy control system as shown in Fig. 1, where

$$
G(s)=\frac{1}{(0.2 s+1)(0.1 s+1)(0.05 s+1)} .
$$

The FLC consists of the following two rules:

$$
\begin{aligned}
& \text { If } e \text { is } M_{1} \text {, then } u_{1}=8.2 e, \\
& \text { If } e \text { is } M_{2} \text {, then } u_{2}=10 e,
\end{aligned}
$$

where $M_{1}$ and $M_{2}$ are the fuzzy sets, whose membership functions $\mu_{1}$ and $\mu_{2}$ are given in (22) and (23) as well as in Fig. 3, where $a=\frac{2}{3}$, and $b=\frac{1}{3}$.

$$
\begin{aligned}
& \mu_{1}(e)=\left\{\begin{array}{c}
0, e<-1 \\
\frac{3}{2}(e+1),-1 \leq e<-\frac{1}{3} \\
1,-\frac{1}{3} \leq e<\frac{1}{3} \\
-\frac{3}{2}(e-1), \frac{1}{3} \leq e<1 \\
0, e \geq 1
\end{array}\right. \\
& \mu_{2}(e)=\left\{\begin{array}{c}
1, e<-1 ; \\
-\frac{3}{2}\left(e+\frac{1}{3}\right),-1 \leq e<-\frac{1}{3} ; \\
0,-\frac{1}{3} \leq e<\frac{1}{3} ; \\
\frac{3}{2}\left(e-\frac{1}{3}\right), \frac{1}{3} \leq e<1 ; \\
1, e \geq 1 .
\end{array}\right.
\end{aligned}
$$

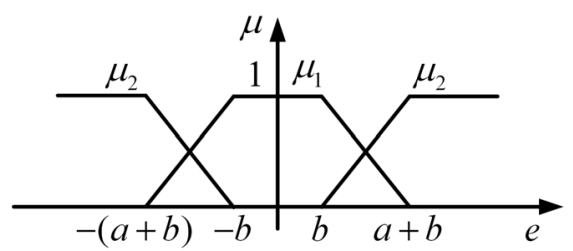

Fig. 3. The membership functions of $M_{1}$ and $M_{2}$.

This T-S fuzzy control system can be represented by the state-space form below:

$$
\left\{\begin{array}{c}
\dot{\boldsymbol{x}}=\boldsymbol{A} \boldsymbol{x}+\boldsymbol{b} \varphi(e), \\
y=\boldsymbol{c x}, \\
e=r-y
\end{array}\right.
$$

where,

$$
\boldsymbol{A}=\left[\begin{array}{ccc}
0 & 1 & 0 \\
0 & 0 & 1 \\
-1000 & -350 & -35
\end{array}\right], \boldsymbol{b}=\left[\begin{array}{l}
0 \\
0 \\
1
\end{array}\right], \boldsymbol{c}^{T}=\left[\begin{array}{c}
1000 \\
0 \\
0
\end{array}\right]
$$

If $r=0$, then $\boldsymbol{x}_{e}=0$ is the equilibrium point of the above T-S fuzzy control system. We can conclude that the equilibrium is globally asymptotically stable by using the circle criterion ${ }^{20}$. However, it is not guaranteed that our T-S fuzzy control system is also Lyapunov stable, when $r$ is chosen to be other constant values. For example, the output in Fig. 4, as $r=8.4$, apparently demonstrates that the T-S fuzzy control system is not asymptotically stable.

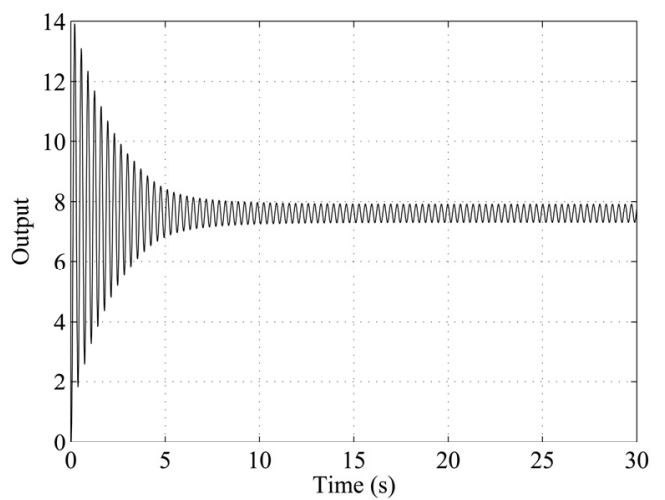

Fig. 4. The output of T-S fuzzy control system with reference input $r=8.4$.

Moreover, we can show that the equilibrium is even unstable. Actually, the equilibrium $\boldsymbol{x}_{e} \approx\left[\begin{array}{llll}0.0076 & 0 & 0\end{array}\right]^{T}$ under the reference input $r=8.4$ can be derived by solving the following equation

$$
\dot{\boldsymbol{x}}_{e}=\boldsymbol{A} \boldsymbol{x}_{e}+\boldsymbol{b} \varphi\left(r-\boldsymbol{c} \boldsymbol{x}_{e}\right)=0 \text {. }
$$

If the closed-loop system is asymptotically stable, $e_{e}=r-c x_{e} \approx 0.8$ is the steady state of the error signal. By linearizing the nonlinearity $\phi(e)$ at $e=0.8$, we have

$$
\left.\dot{\phi}(e)\right|_{e=0.8}=5.4 e+7.3=11.62 .
$$

It is easy to verify that the Nyquist plot of $G(s)$ encircles the point $\left(-\frac{1}{11.62}, 0\right)$ once, which implies that the linearization of this T-S fuzzy control system at the equilibrium point $\boldsymbol{x}_{e}$ is unstable, when $r=8.4$. 
From the above case study, it can be concluded that the asymptotic stability of the T-S fuzzy control system (when $r=0$ ) does not simply imply that the same system is asymptotically stable when $r$ is other constant inputs. Although this example is illustrated by the T-S fuzzy control system, a Mamdani fuzzy control system can also be constructed to demonstrate the similar effects of the reference inputs.

\section{Stability Analysis}

In this section, we use the off-axis circle criterion to analyze the stability of the T-S fuzzy control system in Fig. 1. The main results are summarized in Theorem 2 . We first briefly introduce some important conclusions of Ref. 23.

Theorem 1: Given the T-S fuzzy control system with the fuzzy logic rules (1) in Fig. 1, where $r=0$, and $G(s)$ is the transfer function of the nominal linear plant. Denote $\phi(e)$ as the nonlinear mapping achieved by the T-S fuzzy controller and $\phi(e) \in M_{\left[k_{\min }, k_{\max }\right]}$. This system is globally asymptotically stable, if one of the following three conditions is satisfied:

1). If $0=k_{\min }<k_{\max }, G(s)$ is Hurwitz, and the Nyquist plot of $G(j \omega)$ lies entirely to the right of a straight line passing through the point $\left(-\frac{1}{k_{\max }}, 0\right)$, as $\omega$ varies from 0 to $+\infty$;

2 ). If $0=k_{\min }<k_{\max }$, the Nyquist plot of $G(j \omega)$ encircles the point $\left(-\frac{1}{k_{\max }}, 0\right) \quad m$ times in the counterclockwise direction, as $\omega$ varies from $-\infty$ to $+\infty$, and lies outside an off-axis disk passing through the points $\left(-\frac{1}{k_{\min }}, 0\right)$ and $\left(-\frac{1}{k_{\max }}, 0\right)$, as $\omega$ varies from 0 to $+\infty$, where $m$ is the number of the poles of $G(s)$ with positive real parts.

$3)$. If $k_{\min }<0<k_{\max }, G(s)$ is Hurwitz, and the Nyquist plot of $G(j \omega)$ lies entirely inside an off-axis disk passing through the points $\left(-\frac{1}{k_{\min }}, 0\right)$ and $\left(-\frac{1}{k_{\max }}, 0\right)$, as $\omega$ varies from 0 to $+\infty$.

\section{Proof. Refer to Ref. 23.}

For the T-S fuzzy control system in Fig. 1, different from the circle criterion-based stability conditions, the following novel condition on the basis of the off-axis circle criterion can guarantee the global asymptotic stability, when $r=r_{0}, r_{0} \in R$, if the closed-loop system is proven to be globally asymptotically stable by Theorem 1.

Theorem 2: Suppose the T-S fuzzy control system in Fig. 1 is proven to be globally asymptotically stable by Theorem 1. Then the T-S fuzzy control system is globally asymptotically stable, when $r=r_{0}, r_{0} \in R$.

Proof. The state space equations of the T-S fuzzy control system are given as follows:

$$
\left\{\begin{array}{l}
\dot{\boldsymbol{x}}=\boldsymbol{A} \boldsymbol{x}+\boldsymbol{b} \varphi(e) \\
y=\boldsymbol{c x} \\
e=r-y
\end{array}\right.
$$

Assume that $\boldsymbol{x}_{e}$ and $e_{e}$ are the equilibrium points corresponding to the given reference input $r=r_{0}$, $r_{0} \in R$. With (28), we have

$$
\left\{\begin{array}{l}
\dot{\boldsymbol{x}}_{e}=\boldsymbol{A} \boldsymbol{x}_{e}+\boldsymbol{b} \varphi\left(e_{e}\right)=0, \\
y_{e}=\boldsymbol{c} \boldsymbol{x}_{e}, \\
e_{e}=r-y_{e} .
\end{array}\right.
$$

Subtracting (29) from (28) yields

$$
\left\{\begin{array}{l}
\dot{\bar{x}}=A \overline{\boldsymbol{x}}+\boldsymbol{b} \bar{\varphi}(\bar{e}), \\
\bar{y}=\boldsymbol{c} \overline{\boldsymbol{x}}, \\
\bar{e}=\bar{r}-\bar{y} .
\end{array}\right.
$$

where $\overline{\boldsymbol{x}}=\boldsymbol{x}-\boldsymbol{x}_{e}, \bar{e}=e-e_{3}, \bar{y}=y-y_{e}, \bar{r}=0$, and $\bar{\varphi}(\bar{e})=\varphi\left(\bar{e}+e_{e}\right)-\varphi\left(e_{e}\right)$, whose nonlinearity is shown in Fig. 5. Here, we shift the equilibrium to the origin point, and as a consequence, the reference input can be considered to be zero. From Fig. 5, it is obvious that $\phi(\cdot)$ and $\bar{\phi}(\cdot)$ also belongs to $M_{\left[k_{\min }, k_{\max }\right]}$.

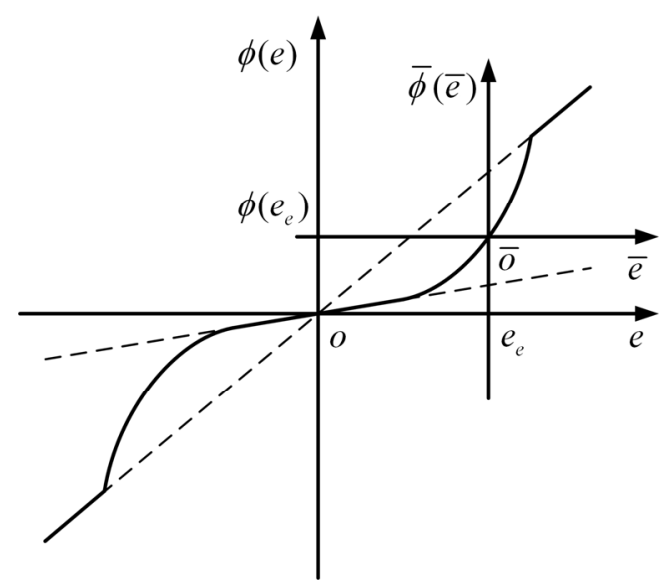

Fig. 5. Nonlinearity of $\bar{\phi}(\cdot)$.

Therefore, it can be concluded that the equilibrium point $\boldsymbol{x}_{e}$ is globally asymptotically stable, if $\overline{\boldsymbol{x}}$ is globally asymptotically stable. In other words, $\boldsymbol{x}_{e}$ and $\overline{\boldsymbol{x}}$ have the same stability property. Since system (30) is globally asymptotically stable by hypothesis, the T-S 
fuzzy control system in Fig. 5 is also globally asymptotically stable, when $r=r_{0}, r_{0} \in R$.

By Theorem 2, the global asymptotic stability of different equilibriums is guaranteed by that of the equilibrium at the origin. The off-axis circle criterion focuses on the global property of the nonlinearity, while the circle criterion and Popov criterion can only handle the local property. Note that the off-axis circle criterion is applicable to those cases, where the prior knowledge of the nonlinearity is known beforehand. As a matter of fact, the stability analysis of different equilibriums can be unified by the off-axis circle criterion.

\section{Simulations}

Example 1. For the T-S fuzzy control system in Section 3 , two proportional controllers $k_{1}=1.73$ and $k_{2}=3.16$ can be obtained by using the Bode plot. We construct a T-S fuzzy controller as follows:

$$
\begin{aligned}
& \text { If } e \text { is } M_{1} \text {, then } u_{1}=1.73 e, \\
& \text { If } e \text { is } M_{2} \text {, then } u_{1}=3.16 e .
\end{aligned}
$$

The membership functions are shown in Fig. 3, where $a=0.6$ and $b=0.4$. By Lemma 1 , we have $\phi(e) \in M_{[1.73,5.544]}$. The Nyquist plot of $G(j \omega)$ with the disk passing through points $\left(-\frac{1}{1.73}, 0\right)$ and $\left(-\frac{1}{5.544}, 0\right)$ is shown in Fig. 6, where the dashed line is the Nyquist plot of $G(j \omega)$, as $\omega$ varies from $-\infty$ to 0 .

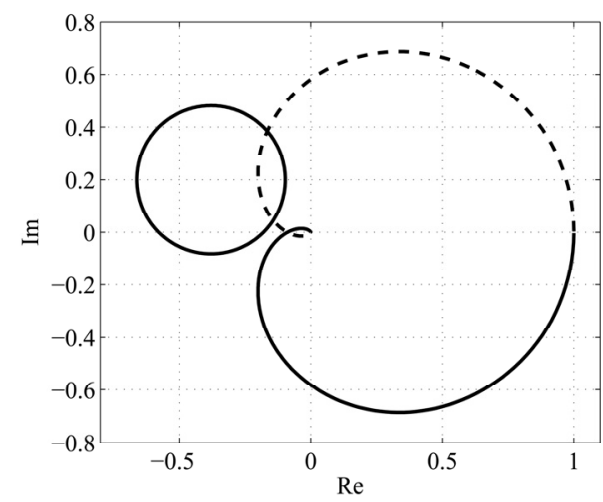

Fig. 6. Nyquist plot of $G(j \omega)$ and off-axis disk passing

$$
\text { through points }\left(-\frac{1}{1.73}, 0\right) \text { and }\left(-\frac{1}{5.544}, 0\right) \text {. }
$$

Apparently, with Theorem 2, the T-S fuzzy control system is globally asymptotically stable for any constant reference input. As an illustrative example, Fig. 7 shows

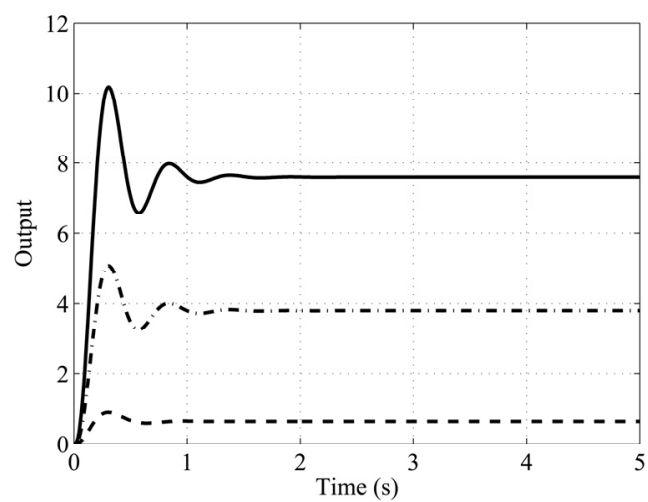

Fig. 7. Responses of T-S fuzzy control system, when $r=1$ (dash line), $r=5$ (dash-dot line), and $r=10$ (solid line).

the responses of the closed-loop system, when $r=1$, $r=5$, and $r=10$.

Example $2{ }^{24}$. Construct an FLC for the T-S fuzzy control system in Fig. 1, where the plant is unstable, and its transfer function is as follows:

$$
G(s)=\frac{2 s+1}{s(s-1)(0.5 s+1)} .
$$

The T-S fuzzy controller is built as in (31) and (32), where $k_{1}=1.25$ and $k_{2}=1.6$. The membership functions of this fuzzy controller are the same as that of Example 1. By Lemma 1, we know that $\phi(e)$ belongs to $M_{[1.25,2,184]}$, and we can find an off-axis disk passing through points $\left(-\frac{1}{1.25}, 0\right)$ and $\left(-\frac{1}{2.184}, 0\right)$ without intersecting or being tangent with the Nyquist plot of $G(j \omega)$, as $\omega$ varies from 0 to $+\infty$ (in Fig. 8). The Nyquist plot also encircles point $\left(-\frac{1}{1.25}, 0\right)$, as $\omega$ varies from $-\infty$ to $+\infty$.

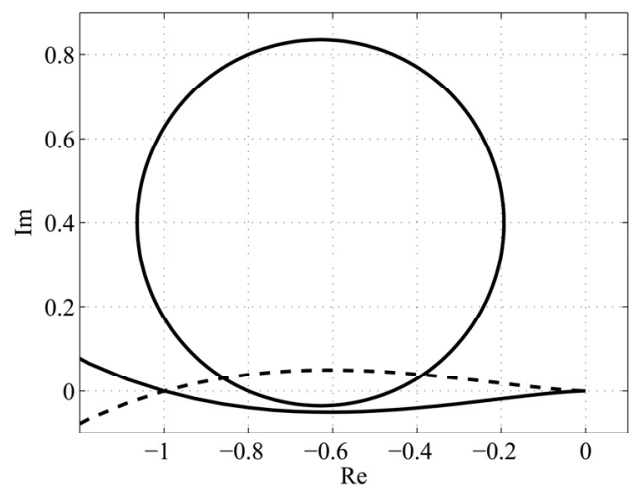

Fig. 8. Nyquist plot of $G(j \omega)$ and off-axis disk passing through points $\left(-\frac{1}{1.25}, 0\right)$ and $\left(-\frac{1}{2.184}, 0\right)$. 
On the basis of Theorem 2, we conclude that our T-S fuzzy control system is asymptotically stable with $k_{1}=1.25$ and $k_{2}=1.6$.

Example 3: In this example, the well-known inverted pendulum problem is used to demonstrate how Theorem 2 can be applied to a nonlinear control system, whose model is given as follows:

$$
\begin{aligned}
& \dot{x}_{1}=x_{2}, \\
& \dot{x}_{2}=\frac{g \sin x_{1}-a m l x_{2}^{2} \sin 2 x_{1} / 2-a \cos x_{1} \cdot u}{4 l / 3-a m l \cos ^{2} x_{1}} .
\end{aligned}
$$

where $x_{1}$ represents the vertical angle of the pendulum, $x_{2}$ is the angular velocity, $g=9.8 \mathrm{~m} / \mathrm{s}^{2}$ is the gravity constant, $m$ is the pendulum mass, $M$ is the cart mass, $l$ is the half length of the pendulum, $u$ is the force exerted on the cart, and $a=\frac{1}{m+M}$. Here, we assume that $M=8.0 \mathrm{~kg}, m=2.0 \mathrm{~kg}$, and $l=0.5 \mathrm{~m}$.

When $x_{1}$ and $x_{2}$ are about zero, we obtain

$$
\begin{aligned}
& \sin x_{1} \approx x_{1}, \\
& \cos x_{1} \approx 1, \\
& x_{2}^{2}(t) \approx 0 .
\end{aligned}
$$

By substituting (35) into (34), we linearize the nonlinear model of the inverted pendulum as

$$
\begin{aligned}
& \dot{x}_{1}=x_{2}, \\
& \dot{x}_{2}=\frac{g x_{1}-a u}{4 l / 3-a m l} .
\end{aligned}
$$

When $x_{1}$ is about $\frac{\pi}{8}$, and $x_{2}$ is about zero, we have:

$$
\begin{aligned}
& \sin x_{1} \approx \frac{8}{\pi} \sin \frac{\pi}{8} \cdot x_{1}, \\
& \cos x_{1} \approx \cos \left(\frac{\pi}{8}\right), \\
& x_{2}^{2}(t) \approx 0 .
\end{aligned}
$$

The linearized model at $x_{1}=\frac{\pi}{8}$ is

$$
\begin{aligned}
& \dot{x}_{1}=x_{2}, \\
& \dot{x}_{2}=\frac{g \frac{8}{\pi} \sin \frac{\pi}{8} \cdot x_{1}-a \cos \frac{\pi}{8} \cdot u}{\frac{4 l}{3}-a m l \cos ^{2} \frac{\pi}{8}} .
\end{aligned}
$$

When $x_{1}$ is about $-\frac{\pi}{8}$, and $x_{2}$ is about zero, the linearized model can be similarly obtained as in (38). Therefore, there are two linearized models. When $x_{1}$ and $x_{2}$ are both about zero, the transfer function is

$$
G_{1}(s)=-\frac{3}{17 s^{2}-294}
$$

When $x_{1}$ is about $\pm \frac{\pi}{8}$, and $x_{2}$ is about zero, the transfer function is

$$
G_{2}(s)=-\frac{0.1589}{s^{2}-16.428} .
$$

To stabilize the inverted pendulum at $x_{1} \approx 0$, an appropriate linear compensator is derived by the Bode plot as follows:

$$
C_{1}(s)=-k_{1} C(s)=\frac{-138\left(\frac{1}{2.7} s+1\right)}{\frac{1}{80} s+1} .
$$

When $x_{1}$ is about $\pm \frac{\pi}{8}$, the compensator is acquired in the same way, which is

$$
C_{2}(s)=-k_{2} C(s)=\frac{-150\left(\frac{1}{2.7} s+1\right)}{\frac{1}{80} s+1} .
$$

If we embed the dynamics of the two compensators into the inverted pendulum, we can acquire a generalized plant as

$$
G(s)=G_{1}(s) C(s)=\frac{3\left(\frac{1}{2.7} s+1\right)}{\left(17 s^{2}-294\right)\left(\frac{1}{80} s+1\right)} .
$$

On the basis of the gains of the linear compensators given above, a T-S fuzzy controller is constructed as

$$
\begin{aligned}
& \text { If } x_{1} \text { is about zero, then } u_{1}=138 \mathrm{e}, \\
& \text { If } x_{1} \text { is about } \pm \frac{\pi}{8} \text {, then } u_{2}=150 \mathrm{e} .
\end{aligned}
$$

The corresponding membership functions are shown in Fig. 3, where $a=\frac{3}{32} \pi$, and $b=\frac{\pi}{32}$. By Lemma 1, we have $\varphi(e) \in M_{[138,166]}$. The Nyquist plot of $G(j \omega)$ is given in Fig. 9, from which we can conclude that this inverted pendulum system is indeed asymptotically stable. For example, when $x_{1}(0)=8^{\circ}$, the stable system response is illustrated in Fig. 10.

\section{Conclusions}

In this paper, we present a sufficient stability condition for a type of T-S fuzzy control systems by using the offaxis circle criterion. Different from other frequency domain-based approaches, it can guarantee the global 


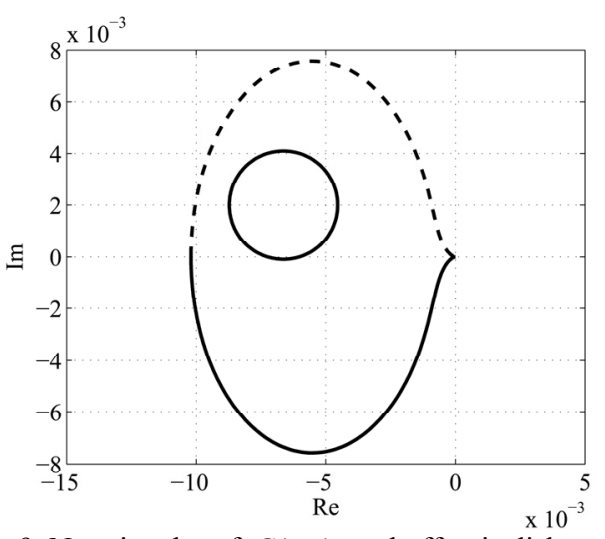

Fig. 9. Nyquist plot of $G(j \omega)$ and off-axis disk passing through points $\left(-\frac{1}{138}, 0\right)$ and $\left(-\frac{1}{166}, 0\right)$.

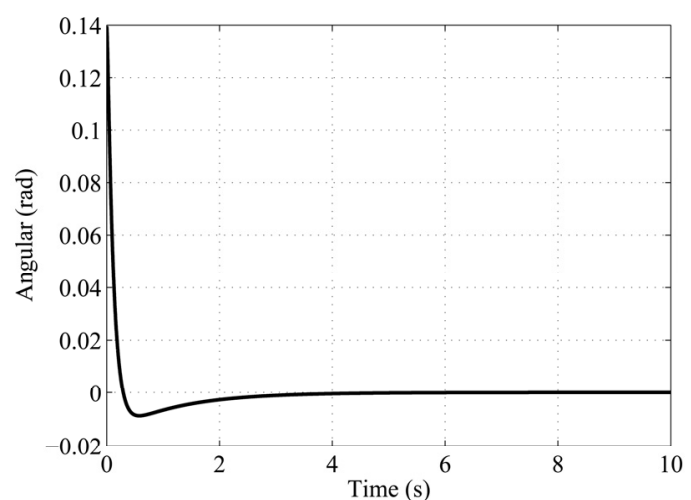

Fig. 10. The angular trajectory of inverted pendulum with initial angle of $8^{\circ} \approx 0.1396 \mathrm{rad}$.

asymptotic stability in case of various constant reference inputs. The proposed method has also a simple graphical interpretation. Three simulation examples have been given to demonstrate its efficiency. In the future work, we are going to generalize our stability analysis technique to the fuzzy control systems with interval plants.

\section{Acknowledgements}

This research work was funded by the NSFC under Grant No. 60874084 and the Academy of Finland under Grants 135225, 127299, and 137837. X. Z. Gao's research is also supported by the Program for Professor of Special Appointment (Eastern Scholar) at Shanghai Institutions of Higher Learning.

\section{References}

1. T. Takagi and M. Sugeno, Fuzzy identification of system and its applications to modeling and control, IEEE
Transactions on Systems, Man, and Cybernetics 15(1) (1985) 116-132.

2. M. Sugeno and G. T. Kang, Fuzzy modeling and control of multilayer incinerator, Fuzzy Sets and Systems 18(3) (1986) 329-346.

3. K. Tanaka and M. Sugeno, Stability analysis and design of fuzzy control systems, Fuzzy Sets and Systems 45(2) (1992) 135-156.

4. M. Johansson, A. Rantzer, and K. E. Årzén, Piecewise quadratic stability of fuzzy systems, IEEE Transactions on Fuzzy Systems 7(6) (1999) 713-722.

5. G. Feng, A survey on analysis and design of model-based fuzzy control systems, IEEE Transactions on Fuzzy Systems 14(5) (2006) 676-697.

6. K. Tanaka and H. O. Wang, Fuzzy Control Systems Design and Analysis: A Linear Matrix Inequality Approach (John Wiley \& Sons, Inc., New York, 2001).

7. S. Zhou, G. Feng, J. Lam, and S. Xu, Robust $H_{\infty}$ control for discrete-time fuzzy systems via bias-dependent Lyapunov functions, Information Sciences 174(3-4) (2005) 197-217.

8. H. K. Lam and F. H. F. Leung, Stability analysis of fuzzy control systems subject to uncertain grades of membership, IEEE Transactions on Systems, Man, and Cybernetics 35(6) (2005) 1322-1325.

9. W. J. M. Kickert and E. H. Mamdani, Analysis of a fuzzy logic controller, Fuzzy Sets and Systems (1978) 29-44.

10. A. Javier and G. Francisco, Describing function method for stability analysis of PD and PI fuzzy controllers, Fuzzy Sets and Systems 143(2) (2004) 233-249.

11. K. S. Ray and D. D. Majumder, Application of circle criteria for stability analysis of linear SISO and MIMO systems associated with fuzzy logic controller, IEEE Transactions on Systems, Man, and Cybernetics 14(1984) 345-349.

12. K. S. Ray, A. M. Ghosh, and D. D. Majumder, -stability and the related design concept for SISO linear system associated with fuzzy logic controller, IEEE Transactions on Systems, Man, Cybernetics 14(1984) 932-939.

13. K. M. Passino and S.Yurkovich, Fuzzy Control (AddisonWesley, Inc., 2001).

14. B. Choi, S. Kwak, and H. Wang, Stability analysis of a simple-structured fuzzy logic controller, Journal of Intelligent \& Robotic Systems 42(2005) 169-178.

15. R. E. Guerra, G. Schmitt-Braess, R. H. Haber, A. Alique, and J. R. Alique, Using circle criteria for verifying asymptotic stability in PI-Like fuzzy control systems: application to the milling process, IEE ProceedingsControl Theory and Applications 150(2003) 619-627.

16. F. Cuesta, F. Gordillo, J. Aracil, and A. Ollero, Stability analysis of nonlinear multivariable Takagi-Sugeno fuzzy control systems, IEEE Transactions on Fuzzy Systems 7(5) (1999) 508-520.

17. F. Cuesta and A. Ollero, Fuzzy control of reactive navigation with stability analysis based on conicity and Lyapunov theory, Control Engineering Practice 12(5) (2004) 625-638. 
18. H. Kang, C. Kwon, H. Lee, and M. Park, Robust stability analysis and design method for the fuzzy feedback linearization regulator, IEEE Transactions on Fuzzy Systems 6(4) (1998) 464-472.

19. C. Park and H. Kang, Graphical and numerical approach to robust stability analysis of fuzzy modeled systems with parametric uncertainty and disturbance, Fuzzy Sets and Systems 151(1) (2005) 99-117.

20. X. Ban, X. Z. Gao, X. Huang, and A. V. Vasilakos, Stability analysis of the simplest Takagi-Sugeno fuzzy control system using circle criterion, Information Sciences 177(20) (2007) 4387-4409.

21. X. Ban, X. Z. Gao, and X. Huang, $L_{2}$-stability analysis of proportional Takagi-Sugeno fuzzy controller based control systems, Intelligent Automation and Soft Computing 16(1) (2010) 61-71.

22. Y. S. Cho and K. S. Narendra, An off-axis circle criterion for the stability of feedback systems with a monotonic nonlinearity, IEEE Transactions on Automatic Control 13 (1968) 413-416.

23. K. R. Cao, X. Z. Gao, X. J. Ban, and X. L. Huang, Stability analysis for a type of proportional T-S fuzzy control systems using the off-axis circle criterion, in Proceedings of 2010 IEEE International Conference on Fuzzy Systems (Spain, Barcelona, 2010), pp. 2879-2884.

24. X. L. Huang, K. R. Cao, X. J. Ban, and X. Z. Gao, Some further results on the stability analysis of a type of T-S fuzzy control systems in the frequency domain, ICIC Express Letters 3(4) (2010) 659-666. 